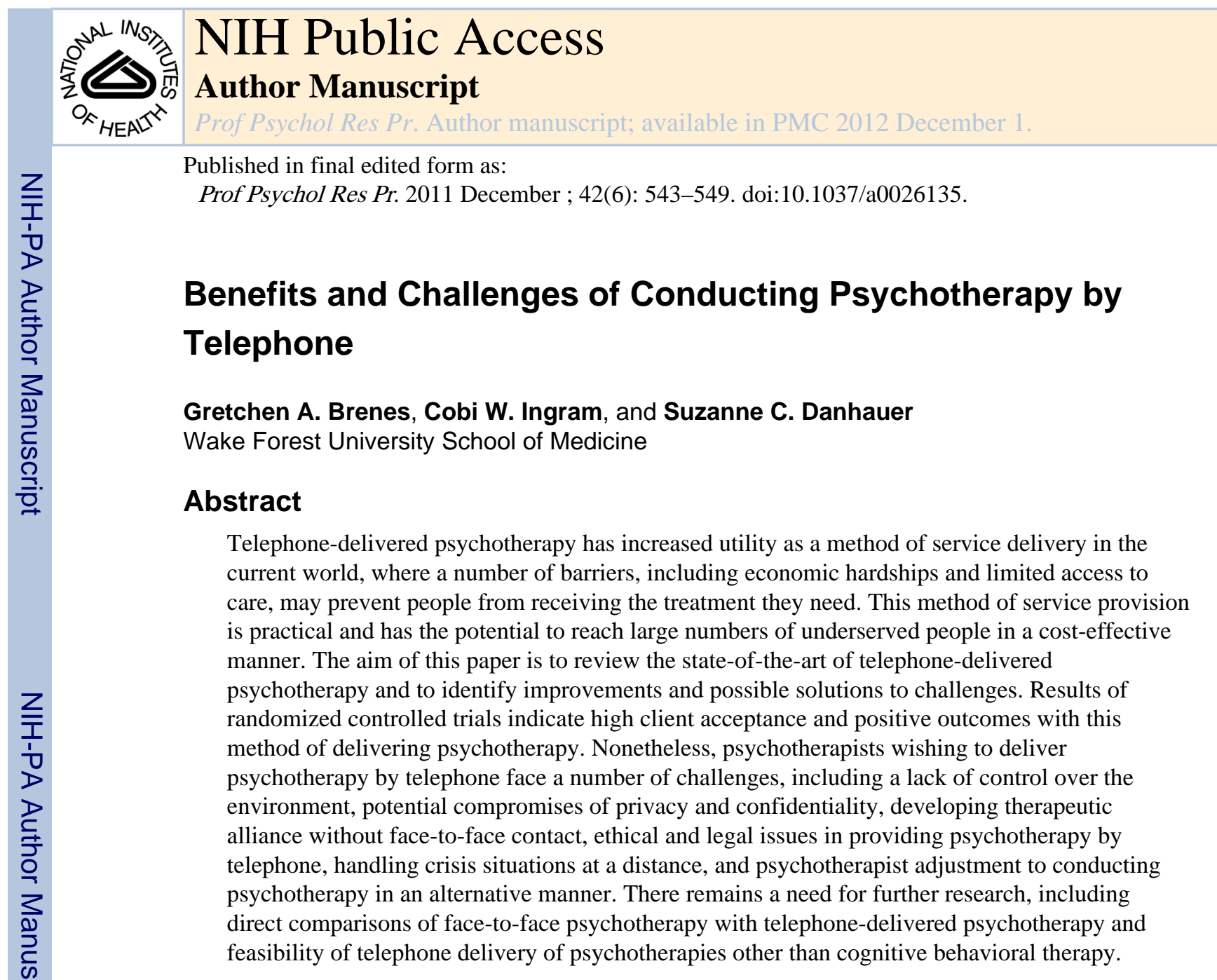

\title{
Keywords
}

telehealth; telephone; psychotherapy; mental health; healthcare access

Correspondence concerning this article should be addressed to Gretchen A. Brenes, Department of Psychiatry and Behavioral Medicine, Wake Forest School of Medicine, Winston-Salem, NC 27157. gbrenes@ wakehealth.edu.

Publisher's Disclaimer: The following manuscript is the final accepted manuscript. It has not been subjected to the final copyediting, fact-checking, and proofreading required for formal publication. It is not the definitive, publisher-authenticated version. The American Psychological Association and its Council of Editors disclaim any responsibility or liabilities for errors or omissions of this manuscript version, any version derived from this manuscript by NIH, or other third parties. The published version is available at www.apa.org/pubs/journals/pro

GRETCHEN A. BRENES received her PhD in clinical psychology from Washington University in St. Louis. She is an associate professor in the Departments of Psychiatry and Behavioral Medicine and Social Sciences and Health Policy at Wake Forest School of Medicine. Her areas of research and practice include late-life anxiety, telephone-delivered psychotherapy, access to mental health services for rural populations, and the effects of anxiety on physical functioning and disability.

COBI W. INGRAM received her MSW from Washington University in St. Louis. She is a Social Worker in the Department of Psychiatry and Behavioral Sciences at Wake Forest School of Medicine. Her areas of professional interest include assessment and treatment of anxiety, therapeutic outcomes, program evaluation, and professional development and training.

SUZANNE C. DANHAUER received her PhD in clinical psychology from the University of Kentucky. She is an associate professor in the Department of Social Sciences and Health Policy in the Division of Public Health Sciences at Wake Forest School of Medicine. Her symptom burden and improve quality of life, psychosocial oncology, and development of various telehealth approaches to intervention delivery, particularly for individuals residing in rural areas. 


\section{Overview of Telehealth}

Telehealth refers to "the use of telecommunications and information technologies to provide access to health information and services across a geographical distance" (Glueckauf, Picket, Ketterson, Loomis, \& Rozensky, 2003, p. 160). Use of telehealth, especially for mental health care, has increased rapidly because of the desire to provide health care to underserved populations, reduce health care costs, and meet consumers' desires (Glueckauf et al., 2003). A number of barriers to traditional face-to-face psychotherapy may prevent people from obtaining the help they need, including transportation issues, need for childcare, perceived stigma, and difficulty leaving work (Bee, Lovell, Lidbetter, Easton, \& Gask, 2010; Mohr et al., 2005). Over two thirds of depressed patients in a primary care setting reported at least one practical barrier (e.g., transportation difficulties) that hampered their ability to attend psychotherapy regularly (Mohr et al. 2006). Telephone-delivered psychotherapy can minimize such barriers, either as the primary method of delivering psychotherapy or as an adjunct to face-to-face psychotherapy.

Telephone-delivered psychotherapy offers increased client convenience with respect to location and flexible timing of appointments (Simon, Ludman, Tutty, Operskalski, \& Von Korff, 2004; Tutty, Ludman, \& Simon, 2005; Tutty, Spangler, Poppleton, Ludman, \& Simon, 2010). Clients can complete sessions at home in privacy, which may reduce concerns about stigma related to mental health treatment (Mozer, Franklin, \& Rose, 2008; Simon et al., 2004) and potentially be less threatening than face-to-face sessions for some clients.

Clients who could benefit from improved access to psychotherapy include older adults, individuals who lack local service providers (especially rural residents), homebound individuals or those with chronic medical illnesses, active military personnel who may prefer care from military providers, and incarcerated clients (Glueckauf et al., 2003). In essence, psychotherapy by telephone makes treatment available to people who may not otherwise receive mental health care (Mohr et al., 2010; Simon et al., 2004).

Despite recent advances in video and computer technology, the telephone may still be a preferred method of providing psychotherapy compared with more technologically advanced communication approaches. First, virtually all U.S. households (96\%) have a telephone land line or cell phone (Federal Communications Commission, 2010) compared with only $62 \%$ with internet access (U.S. Census Bureau, 2007). Furthermore, rural residents, who are likely targets of telehealth approaches, have even less internet access than more urban residents (U.S. Department of Commerce, 2010). Second, using the telephone to deliver psychotherapy requires no special and potentially costly equipment. The only added cost is long-distance charges. Third, videoconferencing often involves travel to a centralized location with a video hookup, which is problematic for homebound individuals and those who cannot or are unwilling to drive. Fourth, conducting psychotherapy by telephone has fewer potential technical difficulties than videoconferencing, e.g. poor picture quality or frozen frames. Finally, neither psychotherapists nor clients need instructions on how to use a telephone.

\section{Randomized Controlled Trials of Telephone-delivered Psychotherapy}

Research to date on telephone-delivered psychotherapy has yielded encouraging findings. In this section, we review randomized controlled trials (RCTs) employing telephone-delivered cognitive behavioral therapy (CBT) for participants with depression or anxiety disorders, mainly because the vast majority of studies examining telephone psychotherapy have focused on CBT. 
Lovell and colleagues (Lovell et al., 2006) conducted the only randomized clinical trial to date comparing face-to-face and telephone-delivered psychotherapy. Participants were 72 adults diagnosed with Obsessive Compulsive Disorder recruited from outpatient psychology clinics in the United Kingdom. They received 10 weeks of exposure and response prevention delivered by 2 "trained" psychotherapists who received regular supervision. The first session was conducted face-to-face for all participants. The attrition rate was low (5.5\%); 3 participants dropped out of face-to-face treatment and 1 dropped out of telephone treatment. Results indicated significant declines in both Yale Brown Obsessive Compulsive Checklist (YBOCS) scores and depressive symptom scores from pre to post-intervention, with no significant between-group differences. Further, $77 \%$ of clients in the telephone group, versus $67 \%$ of clients in the face-to-face group, had evidence of clinically significant results (defined as a $\geq 2$ standard deviation reduction in YBOCS score). Thus, this RCT found comparable statistically and clinically significant changes in symptoms and minimal attrition for both face-to-face and telephone psychotherapy.

Other studies have compared telephone-delivered psychotherapy with treatment as usual or information-only conditions. Brenes and colleagues (Brenes et al., in press) recruited 60 adults 60 years and older with Generalized Anxiety Disorder, Panic Disorder, or Anxiety Disorder Not Otherwise Specified based on the Structured Clinical Interview for DSM-IV (SCID). Participants were randomized to 8-10 sessions of telephone-delivered CBT or an information-only condition. Psychotherapists were a doctoral-level clinical psychology trainee and a master's-level social worker. The attrition rate was low (8.3\%), with 4 participants dropping out of the telephone CBT condition and 1 dropping out of the information-only condition. Recipients of telephone-delivered CBT had significantly reduced worry, general anxiety, anxiety sensitivity, and insomnia symptoms compared with participants receiving information only. Reductions in worry were maintained over a 6month period for the CBT group.

Swinson and colleagues (Swinson, Fergus, Cox, \& Wickwire, 1995) compared telephonedelivered CBT with a wait-list control condition. Participants with Panic Disorder with Agoraphobia $(\mathrm{N}=42)$ were recruited through letters to physicians and newspaper advertisements targeting rural Canadians. The CBT group received 8 sessions of exposure therapy, coping strategies, and discussion of catastrophic thinking. The study psychotherapist had 2 years of experience. Attrition was $9.5 \%(n=4)$. Compared with the wait-list comparison group, participants who received CBT evidenced greater improvements in depressive symptoms, anxiety, distress, fear, and avoidance.

Mohr et al. (Mohr et al., 2005) conducted the only study comparing 2 types of telephonedelivered psychotherapies, CBT and emotion-focused therapy, for treating high depressive symptoms in patients with multiple sclerosis. Overall, $89 \%$ of participants had a diagnosis of Major Depressive Disorder or Dysthymia based on the SCID. Participants randomly received either 16 weeks of $\mathrm{CBT}$ or emotion-focused therapy. Psychotherapists were doctoral-level psychologists who received weekly supervision; randomly selected audiotapes of sessions were rated for competency. Attrition rates were comparable ( 3 from CBT and 4 from emotion-focused therapy). The CBT group experienced significantly greater improvements in depressive symptoms and positive affect than the emotion-focused therapy group.

Most recently, Mohr and colleagues (Mohr, Carmody, Erickson, Jin, \& Leader, 2011) compared telephone-delivered CBT with treatment as usual for veterans diagnosed with depression based on the Mini International Neuropsychiatric Interview. Eighty-five participants received either 16 sessions of telephone-delivered CBT or treatment as usual through Veterans Affairs. Psychotherapists were 3 licensed psychologists, and 10\% of 
psychotherapy sessions were rated for competency in delivering the treatment. Most participants completed at least 4 sessions (97.6\%), with $78 \%$ completing all 16 sessions. Although depressive symptoms improved over time, outcomes did not differ significantly according to treatment group. The authors suggest that veterans may have been more refractory to treatment than other patients.

Telephone-delivered psychotherapy has also been incorporated into collaborative care models. Simon, Ludman and colleagues (Ludman, Simon, Tutty, \& Von Korff, 2007; Simon et al., 2004) conducted a large RCT of telephone-delivered psychotherapy within a health maintenance organization (HMO). Participants were $600 \mathrm{HMO}$ members who had recently ( $<30$ days) begun antidepressant treatment prescribed by their primary care physician. Participants were randomized to telephone-delivered CBT with care management for medication adherence, or usual care. CBT consisted of 8 sessions followed by 2-4 booster sessions. Psychotherapists were master's-level psychotherapists with approximately 20 hours of training and weekly supervision; 15 sessions were reviewed for competence and adherence. Attrition was low, with $79 \%$ of the sample completing 8 or more sessions. Compared with usual care, participants receiving telephone-delivered CBT had significantly greater improvement in depressive symptoms, a change which persisted up to 18 months after treatment. Further, participants who received telephone-delivered CBT were significantly more likely to experience a $50 \%$ improvement in depression scores than participants who received usual care.

Finally, a meta-analysis conducted by Mohr and colleagues (Mohr, Vella, Hart, Heckman, \& Simon, 2008) identified 10 studies that compared telephone-delivered psychotherapy with treatment as usual to treat depressive symptoms. These studies have diverse patient populations and medical comorbidities and different outcome measures. Nonetheless, they found a small but significant effect $(\mathrm{d}=.26)$ for telephone-delivered treatment compared with control groups and a large effect $(\mathrm{d}=.82)$ for pre-post analyses of telephone treatment on reducing depressive symptoms. Although this manuscript focuses on RCTs with persons with a clinical diagnosis of anxiety or depression, telephone-delivered psychotherapy also has been used with nontraditional populations, such as those with chronic illnesses (Mohr et al. 2005) and in a group setting (Heckman et al., 2006).

\section{Client Acceptance of Telephone-delivered Psychotherapy}

Client satisfaction with telephone-delivered treatment is high (Bee et al., 2008). Before receiving psychotherapy, potential clients often report interest and willingness to participate in sessions by telephone. In one study of telephone psychotherapy for depression, approximately $66 \%$ of adults beginning treatment for depression were interested in telephone sessions (Tutty et al., 2010). Although some clients initially may be neutral or even skeptical about psychotherapy by telephone, they typically report high satisfaction upon completion of treatment (Bee et al., 2010; Mohr, Hart, \& Marmar, 2006). Lovell (Lovell et al., 2006) assessed client satisfaction on a scale ranging from 0 to 32 . Differences between participants who received telephone psychotherapy $(\mathrm{M}=28.74$, S.D. $=3.6)$ and face-to-face psychotherapy $(\mathrm{M}=29.84$, S.D. $=2.9)$ were not statistically significant. In fact, both were quite high. Simon et al. (2004) found that only $29 \%$ of clients who received usual primary care were "very satisfied" with treatment compared with 59\% who received telephone care management and psychotherapy. Brenes et al. (in press) used the Client Satisfaction Scale with participants who received telephone psychotherapy. They reported high levels of satisfaction, comparable to earlier findings by Stanley et al. (2009) who used face-to-face CBT in a very similar sample of participants with late-life GAD. 
Participants in research studies of telephone psychotherapy often demonstrate lower rates of attrition than in studies of face-to-face psychotherapy (Ludman et al., 2007; Mohr et al., 2005; Simon et al., 2004; Tutty et al., 2010). In a recent meta-analysis (Mohr et al., 2008), the mean attrition rate for RCTs of telephone-delivered psychotherapy for depression was nearly $8 \%$, markedly lower than the mean attrition rate of $47 \%$ in an earlier meta-analysis of face-to-face psychotherapy (Wierzbicki \& Pekarik, 1993). In short, while high client satisfaction and low attrition alone are not reasons to conduct psychotherapy by telephone, these factors - combined with an emerging literature demonstrating consistent and strong outcome data -- warrant further consideration of the utility and benefits of conducting psychotherapy in this manner.

\section{Challenges of Psychotherapy by Telephone}

Despite these advantages, psychologists must consider a number of issues before undertaking telephone psychotherapy. In this section, we discuss some potential challenges. (See Table 1 for a summary.)

\section{Lack of Control over the Environment}

In traditional face-to-face psychotherapy sessions, the psychotherapist has a considerable amount of control over the environment (i.e., office layout, angle of chairs and distance between them, ability to minimize interruptions and what others can hear during sessions, etc.). However, when psychotherapy is conducted via the telephone, psychotherapists can lose a substantial amount of this control. In fact, psychotherapy by telephone may provide a greater balance between interpersonal power and control over the interaction (Haas, Benedict, \& Kobos, 1996). Situations that can compromise participant privacy include the potential for others to eavesdrop on their psychotherapy sessions (Haas et al., 1996). Psychotherapists should set firm boundaries with clients at the outset, to emphasize that sessions conducted by telephone warrant the same consideration (e.g., lack of interruptions, starting sessions on time) from both therapist and client as those conducted in a therapist's office.

\section{Privacy and Confidentiality}

Concerns about client privacy and confidentiality loom larger when employing current digital and mobile technologies than face-to-face psychotherapy. Confidentiality may be threatened via unintended transmission of information through unsecured telephone connections (i.e., mobile telephones; Mozer et al., 2008). One potentially significant issue is unknowingly speaking with someone other than the intended client on the telephone. One solution is to use a password or another way to verify that the person on the telephone line is, in fact, the client (Heron \& Smyth, 2010). Koocher (2007) presents other scenarios worth considering, including the potential for clients to post excerpts of their recorded psychotherapy sessions online and the possibility that virtually any telephone call could be traced and recorded if a client poses a security threat. While these scenarios may be infrequent, it is imperative to consider their consequences as we embark further into the realm of conducting psychotherapy by telephone.

Just as in face-to-face sessions, psychotherapists and clients should fully discuss all planned services and complete a written contract to confirm their mutual agreement to the terms discussed (e.g., access to the psychotherapist, billing/payment, plan for handling emergencies, etc.). It is critical to fully disclose potential limits of confidentiality with clients for therapies that involve use of wireless telephones, telephones with wireless extensions, and cell phones (Koocher, 2007). Psychotherapists should consider using land lines to ensure greater client privacy and confidentiality. 


\section{Development of Therapeutic Alliance}

Therapeutic alliance is important to the success of psychotherapy. It has been conceptualized as the bond between the psychotherapist and client as they work on the mutually agreedupon tasks and goals of psychotherapy (Bordin, 1979). Some have argued that therapeutic alliance may be difficult to develop in a telephone-based relationship. Haas noted a number of limitations with telephone-delivered psychotherapy (Haas et al., 1996). First, therapeutic alliance may be compromised because the psychotherapist may have difficulty understanding the client's experiences without access to nonauditory cues. Second, it may be difficult for the psychotherapist to convey empathy without being seen. Clients may be nonattentive and feel less in control because they cannot see their psychotherapist, or they may perceive telephone psychotherapy as less important and thus become less invested than in face-to-face psychotherapy (Mozer et al., 2008).

Recent evidence, however, suggests that an effective therapeutic alliance can be established in telephone-delivered psychotherapy. As stated earlier, attrition rates for telephone psychotherapy are low (Brenes et al., in press; Mohr et al., 2005) and client satisfaction with telephone-delivered psychotherapy is high (Brenes et al., in press; Tutty et al., 2010), appearing thus far equivalent to face-to-face psychotherapy (Lovell et al., 2006). Both of these findings suggest the presence of therapeutic alliance.

A few studies have examined therapeutic alliance in telephone-delivered psychotherapy. In the Brenes et al. study, both client and psychotherapist reports of therapeutic alliance as measured by the Working Alliance Inventory were high (M therapist $=70.8$, S.D. $=11.9 ; \mathrm{M}$ client $=67.7$, S.D. $=17.6$; WAI range 7-84; Brenes et al., in press). Similarly, Reese, Conoley, and Broussart (2002) found that clients who received telephone-delivered psychotherapy reported a strong bond with their psychotherapist (as measured by the Working Alliance Inventory-Bond Scale; $M=72.14$, S.D. = 9.79, range 12-84) and were satisfied with treatment. In a qualitative study of telephone-delivered CBT, clients reported developing a sense of psychological closeness with their psychotherapist (Bee et al., 2010). Although differences in therapeutic alliance between face-to-face and telephone-delivered psychotherapy have not been examined fully, evidence suggests that a good therapeutic alliance can, in fact, be established in telephone-based psychotherapy.

\section{Ethical and Legal Issues}

The American Psychological Association (APA) does not yet have ethical guidelines for the practice of telepsychology, resulting in great variability in its implementation (Koocher, 2007). The APA does encourage psychologists who practice telepsychology to do so within the scope of the APA Code of Ethics and has indicated that concerns will be addressed on a case-by-case basis (APA, 2002). The Ohio Psychological Association is the first state association to provide specific telepsychology guidelines (Dielman et al., 2009) regarding (1) appropriate use of technology, with the indication that telepsychology may not be appropriate for every client; (2) legal and ethical requirements, particularly the necessity of confirmation that telepsychology is legal in the state in which the psychotherapist practices; (3) informed consent and disclosure, such that informed consent must be obtained, psychotherapists must disclose their level of experience with telepsychology and the limitations of telepsychology, and alternative means of contact should be discussed if telepsychology is unavailable; (4) secure communications and electronic transfer of client information, with an emphasis on secure means to communicate and avoiding personal identifying information when using nonsecure means of communication; (5) access to and storage of communications, with full disclosure of who has access to communications between the psychotherapist and client and how this information will be stored; (6) fees and financial arrangements specified before psychotherapy begins; (7) supervision provided in 
accordance with state licensing board requirements; and (8) assessment issues, such that psychotherapists are knowledgeable of the psychometric properties of tests and inherent limitations with online administration.

The APA conducted a recent review (2010) of state telehealth laws. Of the 22 states with telehealth laws, only three have telehealth laws that apply to psychologists (California, Kentucky, and Vermont), and California actually excludes telephone contact from these laws. A potential legal issue for psychologists practicing telephone-delivered psychotherapy is the location of both the psychotherapist and the client. Most states consider the provision of psychotherapy to occur in the location of both the psychotherapist and the client. Thus, if the psychotherapist is in one state and the client is in another state, the service occurs in two states. As a result, the psychotherapist unknowingly may be practicing without a license. Some states offer a temporary license that allows limited practice in a secondary state. An alternative is to obtain a license in another state if the psychotherapist plans to do a substantial amount of interstate work. Another legal issue is obtaining informed consent for treatment. Just as in face-to-face psychotherapy, the traditional components of informed consent, such as disclosure of how personal health information will be used, exceptions to confidentiality, and the right to withdraw from treatment, must be explained to the client. Additionally, risks specific to telephone-delivered psychotherapy must be discussed.

\section{Crisis Situations}

Some crises that a psychotherapist may face when conducting telephone psychotherapy are suicidal intent, homicidal intent, and worsening symptoms. Although these crises are not unique to telephone psychotherapy, they must be handled differently than in a typical faceto-face therapeutic relationship. For example, a client in crisis may hang up during a session, preventing the psychotherapist from thoroughly assessing risk. Thus, the development of a safety plan from the start to reduce risk of crises, prevent adverse events, and respond appropriately to adverse events is of the utmost importance in telephone psychotherapy. The safety plan should include careful screening for risk of harm to self or others before psychotherapy starts, monitoring for worsening of symptoms during treatment, and having a safety protocol in place if a crisis occurs during treatment (Luxton, Sirotin, \& Mishkind, 2010).

\section{Psychotherapist Adjustment to Telephone Psychotherapy}

Perhaps the largest issue in telephone psychotherapy is the psychotherapist's openness to working in a manner that seems so untraditional (Bee et al., 2010; Richardson, Frueh, Grubaugh, Egede, \& Elhai, 2009). In fact, some resistance from psychotherapists may stem from their "deeply embedded constructs of the therapeutic relationship" (Bee et al., 2008). Some reasons that psychotherapists may perceive telephone psychotherapy as more difficult include: (1) lack of nonauditory cues that can limit the psychotherapist's ability to interpret client experiences, responses, and ambivalence (Haas et al., 1996; Roffman, 2006); (2) distraction, difficulty maintaining attentiveness during the session, and potential for focusing on extraneous tasks (Haas et al., 1996); (3) potential for sessions to become more social or conversational rather than therapeutic (Haas et al., 1996); and (4) concerns about ability to establish an effective therapeutic alliance with the client (Koocher, 2007; Reese \& Stone, 2005; Richardson et al., 2009).

Interestingly, one study found that although psychotherapists employing telephone psychotherapy were initially skeptical, they were quickly able to develop strong, therapeutic relationships with clients and were generally satisfied with conducting psychotherapy in this manner (Mohr et al., 2006). Bee et al. (2010) have reported that a close therapeutic relationship is, in fact, possible even without physical proximity and suggested that face-to- 
face sessions may not necessarily be the "gold standard." At least one researcher has suggested that psychotherapists' levels of focus may actually increase during telephone sessions, when visual cues are removed from the therapeutic situation (Reese et al., 2002). Similarly, although some may believe that audio plus video sessions are superior for psychotherapy sessions than audio alone, prior research has demonstrated no differences in perceptions of comfort and session distractions when comparing face-to-face, video, and audio-only psychotherapy sessions (Hufford, Glueckauf, \& Webb, 1999). In short, the limited research to date suggests that some issues of concern to psychotherapists about conducting psychotherapy by telephone are not as critical as initially believed.

\section{Situations that May Not Be Optimal for Telephone-Delivered Psychotherapy}

Telephone-delivered psychotherapy may not be appropriate for all clients. Clients whose primary problems are relationship-based in nature may benefit more from face-to-face psychotherapy, including clients who present with discordant relationships or difficulty with maintaining high functioning relationships. Other clients may benefit from a combination of face-to-face and telephone psychotherapy. For example, individuals with problems with avoidance may use the telephone as a way to avoid fully confronting situations that provoke strong emotions and resultant avoidance. These clients may benefit from a mixed approach where telephone sessions can help the client develop coping skills and transition to face-toface psychotherapy. Similarly, telephone-delivered psychotherapy sessions may provide psychotherapists with an opportunity to be present in in vivo exposure exercises that otherwise would be impossible to complete in a traditional psychotherapy session. Clients for whom motivation for psychotherapy is low (such as, clients undergoing court-mandated treatment, clients seeking treatment upon pressure from family or friends) may receive minimal benefit from telephone-delivered psychotherapy. More research is needed to determine who might benefit the most from telephone-delivered psychotherapy.

\section{Conclusions}

Psychologists in the 21st century must address a number of issues regarding the provision of mental health care, including barriers to care, greater emphasis on providing care to the underserved, and lowering health care costs. In this paper, we have focused on one method of doing so - providing psychotherapy by telephone. We have reviewed the evidence base for how and why telephone-delivered psychotherapy can be effective and provided practical suggestions for dealing with some of the difficulties that occur with delivering psychotherapy by telephone.

Although more studies are clearly needed, a growing number of methodologically strong studies demonstrate positive outcomes for telephone-delivered psychotherapy. Much of this research was conducted by Mohr, who concluded that "enough positive trials exist for telephone CBT to be considered an empirically supported treatment" (Mohr et al., 2011, p. 264). Nonetheless, we still lack comparisons of telephone-delivered psychotherapy versus face-to-face psychotherapy and evidence-based determinations of which clients might derive the most benefit from telephone-delivered psychotherapy. Furthermore, most published RCTs compared telephone-delivered psychotherapy with information only, treatment as usual, and wait-list comparison groups rather than more active comparison groups.

Moreover, the one study compared face-to-face and telephone deliveries of the same treatment found no significant differences in outcomes (Lovell et al., 2006). Much more work is needed to fully understand potential differential effects for psychotherapy delivery methods (i.e., face-to-face versus telephone).

Another important issue is the dearth of research on telephone-delivered psychotherapy other than CBT. One potential explanation is that CBT may be easier to deliver by telephone 
because it relies more on the development of specific skills and less on the therapeutic relationship as the "principal agent of change" as in more emotion-focused types of therapies (Beckner, Vella, Howard, \& Mohr, 2007; Bee et al., 2010). This notion is partially supported by Beckner et al. (2007), who found greater therapeutic alliance for telephone CBT than telephone emotion-focused supportive therapy. They suggest that in emotionfocused therapy, the lack of physical proximity and nonverbal communication may hamper development of trust needed to explore and understand one's emotional experience.

Because little research has compared various types of psychotherapy conducted by telephone, little can be said about the relative effectiveness of various methods. There are two notable exceptions. One study compared telephone-delivered CBT with telephonedelivered emotion focused therapy. While both types of psychotherapy produced significant declines in depressive symptoms, CBT recipients experienced significantly greater benefits (Mohr et al., 2005). Another study demonstrated that brief telephone-delivered interpersonal therapy was superior to usual care in reducing depressive symptoms in HIV-AIDS patients (Ransom et al., 2008). Thus, some evidence suggests that non-CBT approaches can be efficacious when delivered by telephone. However, more work is needed to explore this area, to determine how much these approaches may need to be adapted for telephone delivery, and the effect of telephone delivery on therapeutic processes central to the psychotherapy being studied.

Another potential reason for the extensive focus on CBT is that it requires relatively minimal adaptations for telephone delivery. In CBT, most techniques traditionally taught face-to-face can be extended to the telephone (except deep breathing and progressive muscle relaxation techniques where visual observation is helpful). However, use of written materials, DVDs, or online videos to demonstrate the exercises may minimize this concern. Conversely, other techniques may be more feasible to conduct via the telephone. For example, a psychotherapist can be fully present during an in vivo exposure experience without being physically present.

Telephone-delivered psychotherapy is one component of telehealth. In addition to psychotherapy delivered by telephone, video, internet, e-mail, and smart phone technology have been incorporated into mental health treatment. Some applications are self-guided, in which clients may have minimal contact with a psychotherapist. However, increased psychotherapist presence has been associated with significantly better outcomes (Spek et al., 2007), suggesting that psychotherapy delivered by telephone or in person may be preferable. Then again, use of technology with minimal psychotherapist contact may be adequate for very highly motivated clients or those with subthreshold symptoms, whereas increased psychotherapist contact (whether by telephone or videoconference) may be preferred for clients with more serious disorders (Newman et al. 2011). Studies of new modalities for delivering telehealth and the degree of psychotherapist contact will help to develop the most efficacious telehealth treatments.

Overall, research suggests that telephone-delivered psychotherapy has the potential to deliver promising results. Psychotherapists must be aware of the challenges of conducting telephone psychotherapy and develop a plan for dealing with these challenges in advance. With the ever-growing reliance on technology, specific training in alternate methods of delivering psychotherapy (e.g. the telephone) should be integrated into clinical training programs to prepare trainees to be competent providers within the rapidly changing landscape of available mental health services. 


\section{Acknowledgments}

This research was supported by National Institute of Mental Health Grants MH065281 and MH083664 to Gretchen A. Brenes.

\section{References}

American Psychological Association. Ethical principles of psychologists and code of conduct. American Psychologist. 2002; 57:1060-1073. doi:10.1037//0003-066X.57.12.1060. [PubMed: 12613157]

American Psychological Association Practice Organization. Telehealth: Legal Basics for Psychologists. 2010. Retrieved from http://www.apapracticecentral.org/

Bee PE, Bower P, Lovell K, Gilbody S, Richards D, Gask L, Roach P. Psychotherapy mediated by remote communication technologies: a meta-analytic review. BMC Psychiatry. 2008; 8:60. 2008 doi:10.1186/1471-244X-8-60. [PubMed: 18647396]

Bee PE, Lovell K, Lidbetter N, Easton K, Gask L. You can't get anything perfect: "User perspectives on the delivery of cognitive behavioural therapy by telephone.”. Social Science \& Medicine. 2010; 71:1308-1315. doi:10.1016/j.socscimed.2010.06.031. [PubMed: 20675026]

Beckner V, Vella L, Howard I, Mohr DD. Alliance in two telephone-administered treatments: relationship with depression and health outcomes. Journal of Consulting \& Clinical Psychology. 2007; 75:508-512. doi:10.1037/022-006X.75.3.508. [PubMed: 17563167]

Bordin ES. The generalizability of the psychoanalytic concept of the working alliance. Psychotherapy: Theory, Research, and Practice. 1979; 16:252-260. doi:10.1037/h0085885.

Brenes GA, Miller ME, Williamson JD, McCall VM, Knudson M, Stanley MA. A Randomized controlled trial of telephone-delivered cognitive-behavioral therapy for late-life anxiety disorders. American Journal of Geriatric Psychiatry. In Press.

Dielman, M.; Drude, K.; Ellenwood, AE.; Heinlen, KT.; Imar, T.; Lichstein, M.; Asch, PS. Telepsychology Guidelines. 2009. Retrieved from Ohio Psychological Association Retrieved from http://www.ohpsych.org/professionalissues.aspx

Federal Communications Commission, Wireline Competition Bureau. Telephone Subscribership in the United States. 2010. Retrieved from http://hraunfoss.fcc.gov/edocs_public/attachmatch/DOC-301241A1.pdf

Glueckauf RL, Picket TC, Ketterson TU, Loomis JS, Rozensky RH. Preparation for the delivery of telehealth services: A self-study framework for expansion of practice. Professional Psychology: Research and Practice. 2003; 34:159-163. doi:10.1207/s15324796abm3201_4.

Haas LJ, Benedict JG, Kobos JC. Psychotherapy by telephone: risks and benefits for psychologists and consumers. Professional Psychology: Research and Practice. 1996; 27:154-160. doi: 10.1037//0735-7028.27.2.154.

Heckman TG, Barcikowski R, Ogles B, Suhr J, Carlson B, Holroyd K, Garske J. A telephonedelivered coping improvement group intervention for milled-aged and older adults living with HIV/AIDS. Annals of Behavioral Medicine. 2006; 32I:27-38. doi:10.1207/s15324796abm3201_4. [PubMed: 16827627]

Heron KE, Smyth JM. Ecological momentary interventions: incorporating mobile technology into psychosocial and health behavior treatments. British Journal of Health Psychology. 2010; 15:1-39. doi:10.1348/135910709X466063. [PubMed: 19646331]

Hufford BJ, Glueckauf RL, Webb PM. Home-based, interactive videoconferencing for adolescents with epilepsy and their families. Rehabilitation Psychology. 1999; 44:176-193. doi: 10.1037//0090-5550.44.2.176.

Koocher GP. Twenty-first century ethical challenges for psychology. American Psychologist. 2007; 62:375-384. doi:10.1037/0003-066X.62.5.375. [PubMed: 17638436]

Lovell K, Cox D, Haddock G, Jones C, Raines D, Garvey R, Hadley S. Telephone administered cognitive behavior therapy for treatment of obsessive compulsive disorder: randomized controlled non-inferiority trial. British Medical Journal. 2006; 333:883. doi:10.1136/bmj.38940.355602.80. [PubMed: 16935946] 
Ludman EJ, Simon GE, Tutty S, Von Korff M. A randomized trial of telephone psychotherapy and pharmacotherapy for depression: continuation and durability of effects. Journal of Consulting and Clinical Psychology. 2007; 75:257-66. doi:10.1037/0022-006X.75.2.257. [PubMed: 17469883]

Luxton DD, Sirotin AP, Mishkind MC. Safety of telemental healthcare delivered to clinically unsupervised settings: a systematic review. Telemedicine and eHealth. 2010; 16:705-711. doi: 10.1089/tmj.2009.0179.

Mohr DC, Carmody T, Erickson L, Jin L, Leader J. Telephone-administered cognitive behavioral therapy for veterans served by community based outpatient clinics. Journal of Consulting and Clinical Psychology. 2011; 79:261-5. doi:10.1037/a0022395. [PubMed: 21299274]

Mohr DC, Hart SL, Howard I, Julin L, Vella L, Catledge C, Feldman MD. Barriers to psychotherapy among depressed and nondepressed primary care patients. Annals of Behavioral Medicine. 2006; 32:254-8. doi:10.1207/s15324796abm3203_12. [PubMed: 17107299]

Mohr DC, Hart SL, Julian L, Catledge C, Honos-Webb L, Vella L, Tasch ET. Telephone-administered psychotherapy for depression. Archives of General Psychiatry. 2005; 62:1007-1014. doi:10.1001/ archpsyc.62.9.1007. [PubMed: 16143732]

Mohr DC, Hart SL, Marmar C. Telephone administered cognitive-behavioral therapy for the treatment of depression in a rural primary care clinic. Cognitive Therapy and Research. 2006; 30:29-37. doi: 10.1007/s10608-006-9006-0.

Mohr DC, Siddique J, Ho J, Duffecy J, Jin L, Fukuo JK. Interest in behavioral and psychological treatments delivered face-to-face, by telephone, and by internet. Annals of Behavioral Medicine. 2010; 40:89-98. doi:10.1007/s12160-010-9203-7. [PubMed: 20652466]

Mohr DC, Vella L, Hart S, Heckman T, Simon G. The effect of telephone-administered psychotherapy on symptoms of depression and attrition: a meta-analysis. Clinical Psychology: Science and Practice. 2008; 15:243-253. doi:10.1111/j.1468-2850.2008.00134.x. [PubMed: 21369344]

Mozer E, Franklin B, Rose J. Psychotherapeutic intervention by telephone. Clinical Interventions in Aging. 2008; 3:391-396. [PubMed: 18686761]

Newman, Szkodny LE, Llera SJ, Przeworski A. A review of technology-assisted self-help and minimal contact therapies for anxiety and depression: Is human contact necessary for therapeutic efficacy? Clinical Psychology Review. 2011; 31I:89-103. doi:10.1016/j.cpr.2010.09.008. [PubMed: 21130939]

Ransom D, Heckman TG, Anderson T, Garske J, Holroyd K, Basta T. Telephone-delivered, interpersonal psychotherapy for HIV-infected rural persons with depression: a pilot trial. Psychiatric Services. 2008; 59:871-877. doi:10.1176/appi.ps.59.8.871. [PubMed: 18678684]

Reese RJ, Conoley CW, Brossart DF. Effectiveness of telephone counseling: a field-based investigation. Journal of Counseling Psychology. 2002; 49:233-242. doi: 10.1037//0022-0167.49.2.233.

Reese CS, Stone S. Therapeutic alliance in face-to-face versus videoconferenced psychotherapy. Professional Psychology: Research and Practice. 2005; 36:649-653. doi: 10.1037/0735-7028.36.6.649.

Richardson LK, Frueh BC, Grubaugh AL, Egede L, Elhai JD. Current directions in videoconferencing tele-mental health research. Clinical Psychology: Science and Practice. 2009; 16:323-338. doi: 10.1111/j.1468-2850.2009.01170.x. [PubMed: 20161010]

Roffman R. Telephone-delivered interventions for people living with HIV/AIDS: guest editorial. AIDS Behavior. 2006; 11:3-4. doi:10.1007/s10461-006-9176-5.

Simon GE, Ludman EJ, Tutty S, Operskalski B, Von Korff M. Telephone psychotherapy and telephone care management for primary care patients starting antidepressant treatment. Journal of the American Medical Association. 2004; 292:935-942. doi:10.1001/jama.292.8.935. [PubMed: 15328325]

Spek V, Cuijpers P, Nyklicek I, Riper H, Keyzer J, Pop V. Internet-based cognitive behaviour therapy for symptoms of depression and anxiety: A meta-analysis. Psychological Medicine. 2007; 37:31928. doi:10.1017/S0033291706008944. [PubMed: 17112400]

Stanley MA, Wilson NL, Novy DM, Rhoades HM, Wagener PD, Greisinger AJ, Kunik ME. Cognitive behavior therapy for generalized anxiety disorder among older adults in primary care: A 
randomized clinical trial. Journal of the American Medical Association. 2009; 301:1460-7. doi: 10.1001/jama.2009.458. [PubMed: 19351943]

Swinson RP, Fergus KD, Cox BJ, Wickwire K. Efficacy of telephone-administered behavioral therapy for panic disorder with agoraphobia. Behavior Research and Therapy. 1995; 33:465-9. doi: 10.1016/0005-7967(94)00061-N.

Tutty S, Ludman EJ, Simon G. Feasibility and acceptability of a telephone psychotherapy program for depressed adults treated in primary care. General Hospital Psychiatry. 2005; 27:400-410. doi: 10.1016/j.genhosppsych.2005.06.009. [PubMed: 16271654]

Tutty S, Spangler DL, Poppleton LE, Ludman EJ, Simon GE. Evaluating the effectiveness of cognitive-behavioral teletherapy in depressed adults. Behavior Therapy. 2010; 41:229-236. doi: 10.1016/j.beth.2009.03.002. [PubMed: 20412887]

U. S. Census Bureau, Population Division, Education and Social Stratification Branch. Computer and Internet Use in the United States: October 2007. 2007. Retrieved from http://www.census.gov/population/www/socdemo/computer/2007.html

U. S. Department of Commerce, National Telecommunications and Information Administration. Digital Nation: 21st Century America's Progress Toward Universal Broadband Internet Access; 2010. Retrieved from http://www.ntia.doc.gov/reports/2010/NTIA_internet_use_report_Feb2010.pdf

Wierzbicki M, Pekarik G. A meta-analysis of psychotherapy dropout. Professional Psychology: Research and Practice. 1993; 24:190-5. doi:10.1037/0735-7028.24.2.190. 


\section{Table 1}

\section{Addressing Challenges of Telephone-Delivered Psychotherapy}

\begin{tabular}{|l|l|}
\hline Challenge & Suggested Ways to Address Challenge \\
\hline $\begin{array}{l}\text { Lack of control over } \\
\text { environment }\end{array}$ & Set boundaries and require privacy at the start of the relationship \\
\hline Privacy/confidentiality & $\begin{array}{l}\text { Complete written informed consent for treatment reviewing service that will be provided and limits of } \\
\text { confidentiality when using electronic communications (e.g., wireless telephones, cell phones); verify that } \\
\text { person on phone is the client }\end{array}$ \\
\hline Therapeutic alliance & $\begin{array}{l}\text { Set expectations for psychotherapy; inform clients that they will have the psychotherapist's undivided } \\
\text { attention; provide picture of psychotherapist; carefully attend to nonverbal cues }\end{array}$ \\
\hline Ethical/legal issues & $\begin{array}{l}\text { Practice only in state in which licensed; review informed consent for treatment prior to the start of } \\
\text { treatment }\end{array}$ \\
\hline Crisis situations & $\begin{array}{l}\text { Exclude patients who are actively suicidal at intake; have knowledge of resources available to clients } \\
\text { within their county/town }\end{array}$ \\
\hline $\begin{array}{l}\text { Psychotherapist adjustment to } \\
\text { telephone psychotherapy }\end{array}$ & $\begin{array}{l}\text { Provide training that focuses on strengthening and modifying reflective listening skills, use of } \\
\text { psychotherapist's voice to convey warmth and emotion, and attentiveness to solely auditory cues from } \\
\text { clients }\end{array}$ \\
\hline
\end{tabular}

